\title{
Pneumococcal nasopharyngeal carriage among children in Brazil prior to the introduction of the 10-valent conjugate vaccine: a culture- and PCR-based survey
}

\author{
H. G. RODRIGUES ${ }^{1}$, T. C. A. PINTO ${ }^{2}$, R. R. BARROS ${ }^{1}$, L. M. TEIXEIRA ${ }^{2}$ AND \\ F. P. G. NEVES ${ }^{1 *}$ \\ ${ }^{1}$ Department of Microbiology and Parasitology, Universidade Federal Fluminense, Niterói, Rio de Janeiro, Brazil \\ ${ }^{2}$ Department of Medical Microbiology, Universidade Federal do Rio de Janeiro, Rio de Janeiro, Brazil
}

Received 25 October 2016; Final revision 6 February 2017; Accepted 9 February 2017;

first published online 7 March 2017

\section{SUMMARY}

We performed two different approaches (broth enrichment step prior to culture (BEC) and PCR (BEPCR)) for detecting Streptococcus pneumoniae from nasopharyngeal specimens collected from 242 children aged $<6$ years attending one hospital $(n=140)$ and one childcare centre $(n=102)$ in a major urban area in Brazil. These specimens were collected immediately before the introduction of the 10-valent pneumococcal conjugate vaccine (PCV10) and the 13-valent vaccine (PCV13) for routine use in Brazil. Results were compared with previous findings obtained with direct culture (DC) on a selective medium. Colonisation prevalence was 58.3\% $(n=141)$, being higher among children attending the childcare centre $(62.7 \%$ vs. $55 \%)$. The culture-based methods (DC and BEC) enabled the detection of S. pneumoniae in $119(49 \cdot 2 \%)$ and $115(47 \cdot 5 \%)$ children, respectively. The PCR-based method (BEPCR) was more sensitive and 137 (56.6\%) carriers were identified. Twenty-six serogroups/serotypes were identified, predominantly 6B, 19F, 14, 6A, 15C and 23F. Multiple colonisation was observed in $13(5 \cdot 4 \%)$ children. The estimated serotypes coverage of available PCVs was $40 \cdot 4 \%$ for the 10 -valent (included in the Brazilian immunisation programme) and $55.8 \%$ for the 13-valent (only available in private clinics). The use of robust approaches to obtain a more realistic insight about the asymptomatic carrier status is of paramount importance to estimate and assess the impact of vaccine implementation. The combination between culture-based and molecular methods constitutes a suitable strategy.

Key words: Colonisation, culture, pneumococcal conjugate vaccines, sequential multiplex PCR, Streptococcus pneumoniae.

\section{INTRODUCTION}

Streptococcus pneumoniae is associated with high rates of morbidity and mortality in childhood, being responsible for severe diseases such as community-

\footnotetext{
* Author for correspondence: F. P. G. Neves, Department of Microbiology and Parasitology, Universidade Federal Fluminense, Niterói, Rio de Janeiro, Brazil.

(Email: fpgneves@vm.uff.br)
}

acquired pneumonia, bacteraemia and meningitis in all countries, with the greatest disease burden in lower income countries [1].

Nasopharyngeal carriage of pneumococci is an important precondition for the development of pneumococcal diseases, and plays an important role in the transmission of the bacteria [2]. The nasopharynx of infants and young children constitutes the main reservoir of this pathogen. Surveillance studies concerning serotype distribution among carriage isolates 
are useful for monitoring vaccination effects [1, 3]. Likewise, data on serotype distribution in the prevaccination era are important to predict and assess the impact of vaccination [1-3].

In 2010, the 10-valent pneumococcal conjugate vaccine (PCV10) was introduced into the Brazilian national immunisation programme free of charge [4] and the 13-valent vaccine (PCV13) was made available in private clinics, replacing the 7 -valent vaccine (PCV7). Due to the large size and the multitude of differences in the country, regarding cultural, socioeconomic and even climatic characteristics, evaluation of the impact on the use of these vaccines needs to be based on regional data obtained from the pre-vaccine era. Furthermore, while different methods have already been proposed to evaluate pneumococcal carriage [5-7], none is universally adopted.

In a study conducted between March and June 2010, we estimated pneumococcal nasopharyngeal carriage among Brazilian children living in a major urban region by using direct culture (DC) on a selective medium, before the implementation of PCV10 and PCV13 vaccination, which occurred from October of the same year [8]. In the present report, we evaluated the use of two additional approaches for detecting $S$. pneumoniae colonisation, based on the broth enrichment of stored nasopharyngeal specimens, and compared the results with those of the DC methodology performed earlier.

\section{METHODS}

\section{Population}

The nasopharyngeal specimens examined in the present work were part of a pneumococcal surveillance study conducted in 2010 [8]. The 242 children enrolled in the study were $<6$ years of age and attendants of one childcare centre (CCC; $n=102)$ or the emergency room of a paediatric hospital $(\mathrm{PH} ; n=140)$. Their median age was 3 years old (interquartile range $1 \cdot 6-4 \cdot 5$ years old) and 129 of them (53.3\%) were male. Six $(2 \cdot 5 \%)$ children had received at least one dose of PCV7.

\section{DC method}

Single nasopharyngeal specimens were collected from each child by using Nylon fiber flocked mini tip swabs, which were immediately placed in cryogenic vials containing $1 \mathrm{ml}$ of skim milk, tryptone, glucose, and glycerol (STGG) transport medium. All specimens were kept on ice and processed within $6 \mathrm{~h}$ after collection by DC on selective medium (Tryptic soy agar containing $5 \%$ sheep blood and $5.0 \mu \mathrm{g} / \mathrm{ml}$ gentamicin) as described previously [8]. Serotypes of pneumococcal isolates were deduced by sequential multiplex PCR designed for Latin America $[9,10]$ and Quellung reaction, with antisera kindly provided by CDC (Centers for Disease Control and Prevention). After DC, nasopharyngeal specimens were stored at $-180{ }^{\circ} \mathrm{C}$.

\section{Evaluation of two additional pneumococcal detection methods: broth enrichment culture (BEC) and broth enrichment multiplex PCR (BEPCR)}

Nasopharyngeal specimens stored at $-180^{\circ} \mathrm{C}$ for 2 years were reprocessed by two additional approaches, based on previously described procedures [5] with a few modifications. After thawing at room temperature, nasopharyngeal specimens were subjected to an enrichment step, which consisted of transferring $200 \mu \mathrm{l}$ of homogenised STGG medium into $2.0 \mathrm{ml}$ of Todd Hewitt broth (Difco Laboratories, Detroit, MI, USA) supplemented with $0.5 \%$ yeast extract (Difco Labs.) and $0.4 \mathrm{ml}$ of rabbit serum. Following incubation for $6 \mathrm{~h}$ at $37^{\circ} \mathrm{C}$ in $5 \% \mathrm{CO}_{2}$-enriched atmosphere, each specimen was tested by two different approaches/methods. The first approach (BEC) was carried out by smearing a $10 \mu$ l loop of enriched broth culture on blood agar plates (Plast Labor, RJ, Brazil). After overnight incubation at $37^{\circ} \mathrm{C}$ in $5 \%$ $\mathrm{CO}_{2}, \alpha$-haemolytic colonies suspected to be $S$. pneumoniae were re-cultured and tested for susceptibility to optochin and bile solubility for species confirmation. Serotypes of pneumococcal isolates were deduced by sequential multiplex PCR $[9,10]$. For the second approach (BEPCR), DNA was extracted from a $200 \mu \mathrm{l}$ aliquot of enriched broth culture, followed by pneumococcal detection (by amplifying the cps $A$ gene) and serotype deduction by all six sequential multiplex PCR.

\section{DNA extraction}

\section{From BEC isolates}

DNA was extracted from pneumococcal isolates by suspending a loopful of overnight growth from a blood agar plate in $300 \mu 1$ of TE (Tris-EDTA) buffer. The homogenised suspensions were heated at $100{ }^{\circ} \mathrm{C}$ for $10 \mathrm{~min}$, followed by centrifugation $(14.000 \mathrm{rpm} / 1 \mathrm{~min})$ and were stored at $20^{\circ} \mathrm{C}$ until use. 
Table 1. Detection of pneumococcal nasopharyngeal carriage among 242 children $<6$ years old attending one childcare centre or one paediatric hospital in Brazil by using three distinct methodologies

\begin{tabular}{lllll}
\hline \hline \multirow{2}{*}{ Setting } & \multicolumn{2}{l}{ Detection methodology } & \\
\cline { 2 - 4 } & $\mathrm{DC}^{\mathrm{a}}$ & $\mathrm{BEC}$ & $\mathrm{BEPCR}$ & Total \\
\hline Paediatric hospital $(n=140)$ & $59(42 \cdot 1 \%)$ & $58(41 \cdot 4 \%)$ & $76(54 \cdot 3 \%)^{*}$ & $77(55 \%)$ \\
Childcare centre $(n=102)$ & $60(58 \cdot 8 \%)$ & $57(55 \cdot 9 \%)$ & $61(59 \cdot 8 \%)$ & $64(62 \cdot 7 \%)$ \\
Total $(n=242)$ & $119(49 \cdot 2 \%)$ & $115(47 \cdot 5 \%)$ & $137(56 \cdot 6 \%)$ & $141(58 \cdot 3 \%)$ \\
\hline \hline
\end{tabular}

DC, direct culture on selective medium; BEC, broth enrichment culture; BEPCR, broth enrichment multiplex PCR.

${ }^{\text {a }}$ Previously published data [8].

$* P=0.04$ compared with BEC method.

\section{For use in the BEPCR method}

DNA was extracted from a $200 \mu$ l aliquot of the enriched broth culture with the QIAamp DNA Mini Kit (QIAGEN, Valencia, CA, USA).

\section{Sequential multiplex PCR for serotype deduction}

Serogroups or serotypes were determined by using 30 pairs of primers distributed into six sequential multiplex PCR reactions [10] targeting the following serotypes: (1) $6 \mathrm{~A} / 6 \mathrm{~B} / 6 \mathrm{C} / 6 \mathrm{D}, 9 \mathrm{~V} / 9 \mathrm{~A}, 14,19 \mathrm{~F}$ and $23 \mathrm{~F}$; (2) $3,15 \mathrm{~B} / 15 \mathrm{C}, 17 \mathrm{~F}, 18 \mathrm{~A} / 18 \mathrm{~B} / 18 \mathrm{C} / 18 \mathrm{~F}$ and $19 \mathrm{~A}$; (3) $1,5,7 \mathrm{~F} / 7 \mathrm{~A}, 9 \mathrm{~N} / 9 \mathrm{~L}$ and $16 \mathrm{~F}$; (4) $2,4,8,20$ and $22 \mathrm{~F} /$ $22 \mathrm{~A}$; (5) 7C(7B/40), 10A, $11 \mathrm{~A} / 11 \mathrm{D}, 12 \mathrm{~F} /(12 \mathrm{~A} / 44 / 46)$ and $23 \mathrm{~A}$; and $(6) 15 \mathrm{~A} / 15 \mathrm{~F}, 23 \mathrm{~B}, 34,35 \mathrm{~B}$ and 39.

\section{Statistical analysis}

Differences in the performance and sensitivity among the different methods were assessed using the Fisher's exact test. Statistical significance was defined as a $P$ value $<0 \cdot 05$.

\section{Ethical considerations}

The protocols for collection and informed consent were approved by the Ethics Committee of the College of Medicine at the Universidade Federal Fluminense (CAAE no. 0142.0.258.000-09). Written informed consent was obtained from parents or legal guardians.

\section{RESULTS AND DISCUSSION}

The overall pneumococcal nasopharyngeal colonisation rate was $58 \cdot 3 \%(141 / 242)$, taking into account the results of the three methodologies. Carriers corresponded to $55 \%$ of the children at the PH (77/140) and
$62 \cdot 7 \%$ (64/102) among those at the CCC. The results obtained by each methodology are shown in Table 1 .

Regarding the culture-based methods, DC enabled the detection of pneumococci in eight specimens that were negative by BEC, which in turn detected pneumococci in four DC-negative specimens. Overall, the two methodologies combined identified $123 \quad(50 \cdot 8 \%)$ pneumococcal carriers. The relative lower sensitivity of the BEC methodology may be explained by the storage period of the specimens, leading to the loss of viability of some isolates or by the inhibition of $S$. pneumoniae growth by other microorganisms due to the enrichment step [7]. Despite these divergences, our results indicate that the use of the BEC approach is a good alternative for pneumococcal isolation even after a long period of storage, since the results of both methods were very similar.

The sensitivity of the PCR-based methodology was higher when compared with both culture-based methods, especially among the specimens collected from children attending the $\mathrm{PH}(P=0.04$ compared with BEC method). BEPCR was able to detect pneumococcal carriage in $137(56 \cdot 6 \%)$ children (Table 1). In addition, this approach confirmed the findings of the culture-based methods, except in four cases. Three of them involved cpsA-negative isolates non-typeable (NT) by Quellung reaction and multiplex PCR recovered from children at the CCC. The remaining one was a $\operatorname{cps} A$-positive serogroup 18 isolate identified only by BEC and not by DC in a child at the $\mathrm{PH}$, from which the lack of $c p s A$ and serogroup 18-specific product amplifications in the BEPCR method may be due to a failure in DNA extraction or clean-up procedures, resulting in amplification inhibitions.

Twenty-six distinct serotypes were detected among the 141 colonised subjects (Fig. 1; Table 2) and the serotype distribution was similar to that previously observed [9]. Serotypes 6B, 19F, 14, 6A, 15C and 23F 


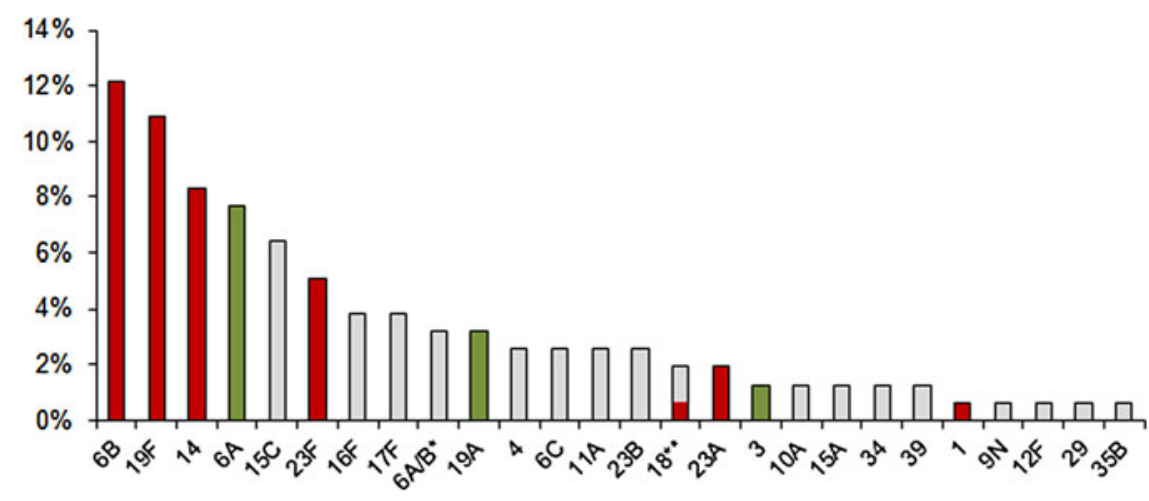

Fig. 1. Serotypes distribution among 156 pneumococci detected in the nasopharynx of 141 children and the estimated serotypes coverage of the PCVs. *Including five serogroup 6 isolates not differentiated between serotype 6A (PCV13 serotype) and 6B (PCV10 and PCV13 serotype) by either the BEC or the BEPCR methods. **Including one 18B isolate, one $18 \mathrm{C}$ isolate (vaccine serotype) and one isolate identified only as serogroup $18 .{ }^{* * *} \mathrm{NT}$, non-typeable pneumococci.

were the most common, with $6 \mathrm{~B}$ predominating among children at the CCC and 19F among those at the PH. Two of the six children who received at least one dose of PCV7 were colonised with serotypes 6B (PCV7 serotype) or $15 \mathrm{C}$ (non-PCV serotype), detected by all the three methods. Although a higher number of carriers has been identified by the BEPCR method (141 vs. 123 from culture-based methods), only one additional serotype was detected. This corresponded to serotype 1 , which is rarely associated with nasopharyngeal colonisation, but frequent among pneumococcal invasive diseases in Latin American and several other low- and high-income countries [11-14].

Multiple colonisation by two to four serotypes was found in 5.4\% (13/242) of the children. In nine cases, co-colonisation was detected by BEPCR, followed by DC with two cases. The remaining two cases were identified by the combination of BEPCR with at least two methods. The combinations were $6+14$ $+16 \mathrm{~F}+23 \mathrm{~F}$ (one child), $14+19 \mathrm{~A}+19 \mathrm{~F}$ (one), $4+$ $6 \mathrm{~A}$ (two), $1+6$ (one), $4+23 \mathrm{~F}$ (one), $6+14$ (one), 6 $+23 \mathrm{~B}$ (one), $6 \mathrm{~B}+\mathrm{NT}$ (one) $14+16 \mathrm{~F}$ (one), $16 \mathrm{~F}+39$ (one), 19A + NT (one) and 23B + 23F (one). The identification of multiple serotypes in a single specimen is a difficult and laborious task, especially because pneumococcal isolates usually exhibit similar colony phenotypes in culture-based methods [15]. However, serogroup 6 and serotype 14 were frequently co-detected in carriage specimens in Brazil [16] and this fact is probably due to their high prevalence in our region prior to the introduction of PCVs for routine use.

It has already been reported that some primers thought to be serogroup/serotype specific, such as those for serogroup 18 and serotype 2, cross-reacted with other unspecific targets and their use to detect $S$. pneumoniae directly from nasopharyngeal swabs should require careful interpretation of the results [17]. In the present study, serogroup 18 and serotype 2 were respectively detected in two and four nasopharyngeal specimens by the BEPCR methodology. Serogroup 18 results were corroborated by the culturebased methodologies. However, serotype 2 was only detected from nasopharyngeal specimens by BEPCR and always associated with another serotype, being, therefore, excluded of the analysis.

Overall, the three approaches allowed for the detection of 156 pneumococcal isolates and 138 (88.5\%) had their capsular type determined. Among the 18 pneumococci which could not be serotyped by the methods we used, three were considered NT isolates, since they did not amplify the cpsA gene and were not serotyped by the Quellung reaction; their detection was only possible by the culture-based methods, mainly DC (Table 2). The remaining 15 cases were identified by BEPCR through cpsA amplification, but no serotype-specific amplification was observed.

It is noteworthy that, beyond 18 cases of unsuccessful serotype determination, the sequential multiplex PCR scheme failed to resolve serogroups or serotypes in six additional isolates detected by the culture-based methods, which could only be serotyped by the Quellung reaction. They included serotypes 19F (three isolates), 6A, 10A and 29 (one isolate each) (Table 2). In multiplex PCR, they exhibited only the cpsA pneumococcal identification control. There are no primers available for serotype 29 detection and, for the other serotypes, some variability in the primers annealing regions can occur. However, using the first multiplex reaction, serotypes or serogroups were 
Table 2. Serotype distribution of 156 pneumococci according to the methodology of pneumococcal serotype determination

\begin{tabular}{|c|c|c|c|}
\hline Serotype (n) & $\mathrm{DC}(n)$ & $\operatorname{BEC}(n)$ & $\operatorname{BEPCR}(n)$ \\
\hline 6B (19) & 6B (19) & $6(18)$ & $6(15), \operatorname{cps} A+(3)$ \\
\hline $19 \mathrm{~F}(17)$ & $19 \mathrm{~F}(15)^{\mathrm{a}}$ & $19 \mathrm{~F}(10), c p s A+(4)$ & $19 \mathrm{~F}(8), c p s A+(9)$ \\
\hline $14(13)$ & $14(11)$ & $14(11)$ & $14(13)$ \\
\hline 6A (12) & $6 \mathrm{~A}(12)^{\mathrm{b}}$ & $6(11), \operatorname{cps} A+(1)$ & $6(9), \operatorname{cps} A+(3)$ \\
\hline $15 \mathrm{C}(10)$ & $15 \mathrm{C}(10)$ & 15B/C (8), cps $A+(1)$ & $15 \mathrm{~B} / \mathrm{C}(4), c p s A+(6)$ \\
\hline $23 \mathrm{~F}(8)$ & $23 \mathrm{~F}(7)$ & $23 \mathrm{~F}(7)$ & $23 \mathrm{~F}(8)$ \\
\hline $16 \mathrm{~F}(6)$ & $16 \mathrm{~F}(4)$ & $16 \mathrm{~F}(4)$ & $16 \mathrm{~F}(6)$ \\
\hline $17 \mathrm{~F}(6)$ & $17 \mathrm{~F}(6)$ & $17 \mathrm{~F}(5), c p s A+(1)$ & $17 \mathrm{~F}(4), c p s A+(2)$ \\
\hline $6(5)^{\mathrm{c}}$ & - & $6(2)$ & $6(5)$ \\
\hline 19A (5) & $19 \mathrm{~A}(5)^{\mathrm{d}}$ & 19A (3), cpsA- (1) & 19A (2), cpsA+ (2) \\
\hline $4(4)$ & $4(1)$ & $4(1)$ & $4(4)$ \\
\hline 6C (4) & $6 \mathrm{C}(4)$ & $6(3), c p s A+(1)$ & $6(1), \operatorname{cps} A+(3)$ \\
\hline $11 \mathrm{~A}(4)$ & $11 \mathrm{~A} \mathrm{(4)}$ & $11 \mathrm{~A} / \mathrm{D}(4)$ & $11 \mathrm{~A} / \mathrm{D}(2), c p s A+(2)$ \\
\hline 23B (4) & 23B (3) & 23B (1) & $23 \mathrm{~B}(3), \mathrm{cps} A+(1)$ \\
\hline $18(3)$ & $18 \mathrm{~B}(1), 18 \mathrm{C}(1)$ & $18(3)$ & $18(2)$ \\
\hline $23 \mathrm{~A}(3)$ & $23 \mathrm{~A}(3)$ & $23 \mathrm{~A}(3)$ & $23 \mathrm{~A}(1), c p s A+(2)$ \\
\hline $3(2)$ & $3(1)$ & $3(1)$ & $3(1), \operatorname{cps} A+(1)$ \\
\hline $10 \mathrm{~A}(2)$ & $10 \mathrm{~A}(2)$ & $\operatorname{cps} A+(2)$ & $\operatorname{cps} A+(2)$ \\
\hline $15 \mathrm{~A}(2)$ & $15 \mathrm{~A}(2)$ & $15 \mathrm{~A} / \mathrm{F}(2)$ & $15 \mathrm{~A} / \mathrm{F}(2)$ \\
\hline $34(2)$ & $34(2)$ & $34(1)$ & $34(2)$ \\
\hline 39 (2) & $39(1)$ & $39(1)$ & $39(2)$ \\
\hline $1(1)$ & - & - & $1(1)$ \\
\hline $9 \mathrm{~N}(1)$ & $9 \mathrm{~N}(1)$ & 9N/L (1) & 9N/L (1) \\
\hline $12 \mathrm{~F}(1)$ & $12 \mathrm{~F}(1)$ & $12 \mathrm{~F}(1)$ & $12 \mathrm{~F}(1)$ \\
\hline $29(1)$ & $29(1)^{e}$ & $c p s A+(1)$ & $\operatorname{cps} A+(1)$ \\
\hline 35B (1) & 35B (1) & 35B (1) & $35 \mathrm{~B}(1)$ \\
\hline Only cps $A+(15)$ & - & $\operatorname{cps} A+(2)$ & $\operatorname{cps} A+(15)$ \\
\hline $\operatorname{cps} A-\mathrm{NT}(3)^{\mathrm{f}}$ & $\operatorname{cps} A-(3)$ & $\operatorname{cps} A-(1)$ & - \\
\hline
\end{tabular}

BEC, broth enrichment culture; BEPCR, broth enrichment multiplex PCR; DC, direct culture on selective medium; $n$, total number of pneumococci that were detected for a particular serotype; NT, non-typeable.

${ }^{\text {a }}$ Three cpsA-positive isolates were identified only by Quellung reaction as serotype $19 \mathrm{~F}$.

${ }^{\mathrm{b}}$ One $\operatorname{cps} A$-positive isolate was identified only by Quellung reaction as serotype 6A.

${ }^{\mathrm{c}}$ Serogroup 6 includes isolates that are not differentiated between serotypes $6 \mathrm{~A}$ and $6 \mathrm{~B}$ by the multiplex PCR used in both the $\mathrm{BEC}$ and the BEPCR methods.

${ }^{\mathrm{d}}$ One cpsA-negative isolate was identified only by Quellung reaction as serotype 19A.

${ }^{\mathrm{e}} \mathrm{A}$ cpsA-positive isolate identified only by Quellung reaction as serotype 29.

${ }^{\mathrm{f}} \mathrm{NT}$ pneumococci by Quellung reaction and multiplex PCR.

deduced for $50 \%(78 / 156)$ of the pneumococci investigated in the present study.

Seven of the 10 serotypes included in PCV10 were detected in our study, corresponding to 63 pneumococcal isolates. This result indicates that PCV10 would have a theoretical impact in reducing asymptomatic carriage by $40 \cdot 4 \%$. Regarding PCV13, which contains serotypes 3, 6A and 19A in addition to those provided in PCV10, this projected impact would be $55.8 \%$ (Fig. 1). On the other hand, although widespread use of PCV is consistently followed by significant reduction in both prevalence of carriage and incidence of invasive diseases caused by vaccine serotypes, several studies have been reporting on the emergence of non-vaccine serotypes, a phenomenon called serotype replacement [18-21]. Therefore, it is highly likely that, while these vaccine serotypes would eventually be almost eliminated, there would be little or no effect to the overall prevalence of pneumococcal carriage.

Our results should be interpreted in the context of several limitations. First, the culture-based methods were performed 2 years apart, making it difficult to compare their efficiency. Second, despite the higher sensitivity of culture-independent approaches, molecular methods can be subjected to confounding, especially when used directly to analyse carriage specimens [17, 22, 23]. 
Previous studies reported on the considerable variation and false positivity when using multiplex PCR [22, 23], yet many findings of the BEPCR method were confirmed by the culture methodologies. Moreover, other technologies, such as microarray and real-time PCR, are more sensitive $[22,24]$ and the combination of microarray with a culture amplification step was identified as one of the best methods to serotype pneumococci in carriage studies [22]. Nonetheless, the use of such technologies in resource-limited settings is very restricted. Finally, it has previously been reported that cpsA is not present in all encapsulated/typeable S. pneumoniae [25] and the prevalence of colonisation may be underestimated. Therefore, the use of a more highly specific and sensitive target in the BEPCR, such as lytA [26], might enhance the detection of pneumococci, including those $c p s A$-negative NT (acapsular) isolates.

In conclusion, the PCR-based approach was more sensitive for detection of pneumococcal colonisation by one or multiple serotypes, despite the limitation to determine the capsular type of some isolates and to detect NT strains. The ongoing tracking of asymptomatic carriers is a valuable tool to estimate and assess the impact of pneumococcal vaccine strategies, since pneumococcal colonisation is considered to reflect the circulating strains/serotypes responsible for diseases in the population and represents a precondition for their development. This is of paramount importance in Brazil since PCV10 was introduced for routine use in the area investigated a few months after the collection of the specimens analysed in this study and, currently, it is universally adopted ( $>90 \%$ coverage) [27]. Considering such purposes, our results corroborate earlier findings $[5,22,24]$ indicating that the combination of culture- and molecular-based approaches can improve surveillance studies yielding more robust information.

\section{ACKNOWLEDGEMENTS}

The authors thank the institutions that agreed to participate in this study. This work was supported by Conselho Nacional de Desenvolvimento Científico e Tecnológico (CNPq), Coordenação de Aperfeiçoamento de Pessoal de Nível Superior (CAPES), Fundação de Amparo à Pesquisa do Estado do Rio de Janeiro (FAPERJ), and Pró-Reitoria de Pesquisa, Pós-Graduação e Inovação da Universidade Federal Fluminense (PROPPi/UFF).

\section{DECLARATION OF INTEREST}

None.

\section{REFERENCES}

1. World Health Organization. Weekly epidemiological record: Relevé épidémiologique hebdomadaire. The Weekly Epidemiological Record 2012; 87: 245-252.

2. Simell B, et al. The fundamental link between pneumococcal carriage and disease. Expert Review of Vaccines 2012; 11: 841-855.

3. Weinberger DM, Malley R, Lipsitch M. Serotype replacement in disease after pneumococcal vaccination. Lancet 2011; 378: 1962-1973.

4. Domingues CMAS, et al. Effectiveness of ten-valent pneumococcal conjugate vaccine against invasive pneumococcal disease in Brazil: a matched case-control. The Lancet Respiratory Medicine 2014; 2: 464471.

5. Carvalho MG, et al. Revisiting pneumococcal carriage by use of broth enrichment and PCR techniques for enhanced detection of carriage and serotypes. Journal of Clinical Microbiology 2010; 48: 1611-1618.

6. Ogami M, et al. A comparison of conventional and molecular microbiology in detecting differences in pneumococcal colonization in healthy children and children with upper respiratory illness. European Journal of Pediatrics 2010; 169: 1221-1225.

7. Satzke C, et al. Standard method for detecting upper respiratory carriage of Streptococcus pneumoniae: updated recommendations from the World Health Organization Pneumococcal Carriage Working Group. Vaccine 2014; 32: 165-179.

8. Neves FP, et al. Nasopharyngeal carriage, serotype distribution and antimicrobial resistance of Streptococcus pneumoniae among children from Brazil before the introduction of the 10 -valent conjugate vaccine. $B M C$ Infectious Diseases 2013; 13: 318.

9. Dias CA, et al. Sequential multiplex PCR for determining capsular serotypes of pneumococci recovered from Brazilian children. Journal of Medical Microbiology 2007; 56: 1185-1188.

10. Centers for Disease Control and Prevention Protocol for Multiplex PCR - S. pneumoniae serotyping - clinical specimens - Latin America set. 2010 (http://www.cdc.gov/ ncidod/biotech/files/pcr-Latin-Amer-clinical-specimens. pdf). Accessed 12:01·13.

11. Ritchie ND, Mitchell TJ, Evans TJ. What is different about serotype 1 pneumococci? Future Microbiology 2012; 7: 33-46.

12. Yoshioka CR, et al. Analysis of invasive pneumoniacausing strains of Streptococcus pneumoniae: serotypes and antimicrobial susceptibility. Journal of Pediatrics 2011; 87: 70-75.

13. Isaacman DJ, McIntosh ED, Reinert RR. Burden of invasive pneumococcal disease and serotype distribution among Streptococcus pneumoniae isolates in young children in Europe: impact of the 7-valent pneumococcal conjugate vaccine and considerations for future conjugate vaccines. International Journal of Infectious Diseases 2010; 14: 197-209.

14. Quadros CA. From global to regional: the importance of pneumococcal disease in Latin America. Vaccine 2009; 27: 29-32. 
15. Hare KM, et al. Random colony selection versus colony morphology for detection of multiple pneumococcal serotypes in nasopharyngeal swabs. The Pediatric Infectious Disease Journal 2008; 27: 178-180.

16. Pimenta FC, et al. Serotype and genotype distributions of pneumococcal carriage isolates recovered from Brazilian children attending childcare centers. Journal of Medical Microbiology 2011; 60: 1455-1459.

17. Carvalho MG, et al. Potential nonpneumococcal confounding of PCR-based determination of serotype in carriage. Journal of Clinical Microbiology 2012; 50: 3146-3147.

18. Weinberger DM, et al. Relating pneumococcal carriage among children to disease rates among adults before and after the introduction of conjugate vaccines. American Journal of Epidemiology 2016; 183: 1055-1062.

19. Davis SM, et al. Impact of pneumococcal conjugate vaccines on nasopharyngeal carriage and invasive disease among unvaccinated people: review of evidence on indirect effects. Vaccine 2013; 32: 133-145.

20. Wroe PC, et al. Pneumococcal carriage and antibiotic resistance in young children before 13-valent conjugate vaccine. The Pediatric Infectious Disease Journal 2012; 31: 249-254.

21. Weinberger DM, Malley R, Lipsitch M. Serotype replacement in disease following pneumococcal vaccination: a discussion of the evidence. Lancet 2011; 378: 1962-1973.

22. Satzke C, et al. The PneuCarriage project: a multicentre comparative study to identify the best serotyping methods for examining pneumococcal carriage in vaccine evaluation studies. PLoS Medicine 2015; 12: e1001903.

23. Wyllie AL, et al. Streptococcus pneumoniae in saliva of Dutch primary school children. PLoS ONE 2014; 9: e102045.

24. Azzari C, et al. Realtime PCR is more sensitive than multiplex PCR for diagnosis and serotyping in children with culture negative pneumococcal invasive disease. PLoS ONE 2010; 5: e9282.

25. Rolo D, et al. Disease isolates of Streptococcus pseudopneumoniae and non-typeable $S$. pneumoniae presumptively identified as atypical $S$. pneumoniae in Spain. PLoS ONE 2013; 8: e57047.

26. Carvalho MG, et al. Evaluation and improvement of real-time PCR assays targeting lytA, ply, and psaA genes for detection of pneumococcal DNA. Journal of Clinical Microbiology 2007; 45: 2460-2466.

27. Moreira M, et al. Impact of the introduction of the pneumococcal conjugate vaccine in the Brazilian routine childhood national immunization program. Vaccine 2016; 34: 2766-2778. 